\title{
Schulbeginn für Kinder mit Asthma
}

\section{Anfang September startete wieder der Alltag}

\begin{abstract}
In Zeiten von Corona sind viele Eltern verunsichert und haben offene Fragen, vor allem wenn die Kleinen an Asthma leiden. Wenn man bedenkt, dass jedes zehnte Kind von Asthma betroffen ist, ist es notwendig, Aufklärungsarbeit durch Experten zu leisten. Dass asthmatische Kinder trotz COVID-19 in den Kindergarten oder in die Schule gehen sollen, steht außer Frage. Bis dato gibt es keine Berichte oder Hinweise darauf, dass Asthma und Allergien bei Kindern tatsächlich Risikofaktoren sind.
\end{abstract}

\section{Asthma bronchiale}

Asthma bronchiale ist die häufigste chronische Erkrankung im Kindesund Jugendalter. Jedes zehnte Kind ist davon betroffen. In mindestens $70 \%$ der Fälle bricht die Krankheit vor dem 5. Lebensjahr aus. Allerdings wird Asthma bronchiale bei Kindern häufig übersehen oder zu spät behandelt. Asthma bronchiale ist zwar noch nicht heilbar, es stehen aber zahlreiche medikamentöse und andere therapeutische Hilfen zur Verfügung. Jede Therapie hat das Ziel, dem erkrankten Kind die vollständige Teilnahme am normalen Leben zu ermöglichen.

\section{Normaler Alltag trotz anormaler Zeiten}

COVID-19 beeinflusst unser aller Leben. Das Beibehalten einer gewissen Normalität im Alltag sollte trotzdem - natürlich unter Beachtung sämtlicher Vorsichtsmaßnahmen - so weit wie möglich verfolgt werden. Kindergarten und Schule sind für die Heranwachsenden wichtig. Vor allem auch wegen des strukturierten Tagesablaufs und der sozialen Kontakte.

\section{Medizinische Antworten für Eltern mit Asthma-Kindern}

DieÖsterreichische Lungenunion (ÖLU) hat sich des Themas angenommen und Univ.-Prof. Dr. Zsolt Szépfalusi von der Universitätsklinik für Kinder- und Jugendheilkunde, Medizinische Universität Wien, Fragen zum Verlauf einer COVID-19 Infektion bei Kindern gestellt - zu den Therapieempfehlungen bis hin zur Frage, ob es ratsam ist, dass Kinder mit Asthma weiter in den Kindergarten oder in die Schule gehen (s. Kasten).

\section{Die ÖLU stellt sich vor}

Die Österreichische Lungenunion (ÖLU) ist eine bundesweit aktive Selbsthilfegruppe für Menschen mit Atemwegserkrankungen, Hauterkrankungen und Allergien. Die ÖLU stützt und fördert die Mündigkeit der Betroffenen. Das Ziel: informierte Patientinnen und Patienten sowie eine informierte Mitwelt. Da es der ÖLU ein Anliegen ist, alle Betroffenen zu erreichen, wurden die Videos mit den Experten auch in Serbisch, Türkisch und Englisch übersetzt.

Die ÖLU ist ein gemeinnütziger Verein und wurde bei den Aufklä-

\section{COVID-19 und Kinder mit Asthma}

Gespräch mit Prof. Univ.-Prof. Dr. Zsolt Szépfalusi

Infektionen mit COVID-19 verlaufen bei Kindern meist milde? Trifft das auch auf Kinder mit Asthma zu? Gibt es dazu neue Erkenntnisse?

Szépfalusi: Die Tatsache, dass COVID-19-Infektionen bei Kindern milde verlaufen, hat sich in den letzten Wochen bis Monaten erhärtet, seitdem wir von dieser Erkrankung wissen. Die Daten zu Asthma-Kindern mit COVID-19-Erkrankungen sind sehr, sehr mager. Es gibt also wenige Kinder, die bis jetzt aufgefallen sind, und bei denen ist kein besonders anderer Verlauf beobachtet worden, als bei Kindern ohne Asthmaerkrankung. Sollte ich mein an Asthma erkranktes Kind besonders beobachten? Gibt es spezielle Therapieempfehlungen?

Szépfalusi: Es gibt Empfehlungen, wie man in der Behandlung und Therapie von Patienten mit Asthma umgehen soll, auch speziell von bestimmten Fachgesellschaften. Die Gesellschaft für pädiatrische Pneumologie und die Gesellschaft für pädiatrische Allergologie, die deutschsprachig die Regionen Österreich, Schweiz und Deutschland abdeckt, haben Empfehlungen herausgegeben, keine Änderungen der Therapie vorzunehmen und ganz speziell die erforderlichen Therapiesteigerungen tatsächlich durchzuführen; einschließlich Biologika-Therapien.

Ist es ratsam, dass mein Kind mit Asthma weiter in den Kindergarten oder in die Schule geht? Szépfalusi: Ja, weil Lernen wichtig ist und schon zahlreiche Wochen bis Monate in dieser COVID-Periode verloren gegangen sind. Es gibt wenige Hinweise, dass Kindergärten besonders betroffen sind. Es gibt zwar ein paar Hotspots, aber grundsätzlich ist es für Kinder auch mit Asthma wichtig, in Kindergärten bzw. Schulen zu gehen, natürlich unter der Voraussetzung, dass man die Kontrolle über die Pandemie, also Neuerkrankungen von COVID-19, hat.

Kann ich ohne Angst vor Ansteckung zum Kinderfacharzt gehen?

Szépfalusi: Ja, der erste Anlaufpartner für alle Erkrankungen, die Kinder betreffen, ist der Kinderfacharzt, und das soll auch in der jetzigen Zeit so weiterbestehen, auch wenn der Verdacht auf COVID-19 vorliegt. Kinderärzte sind gerüstet, mit Infektionskrankheiten aller Art korrekt umzugehen, und da gilt es immer, die eine und andere Erkrankung voreinander zu schützen, und dafür sind Kinderärzte gut gerüstet. 


\section{Springer Medizin}

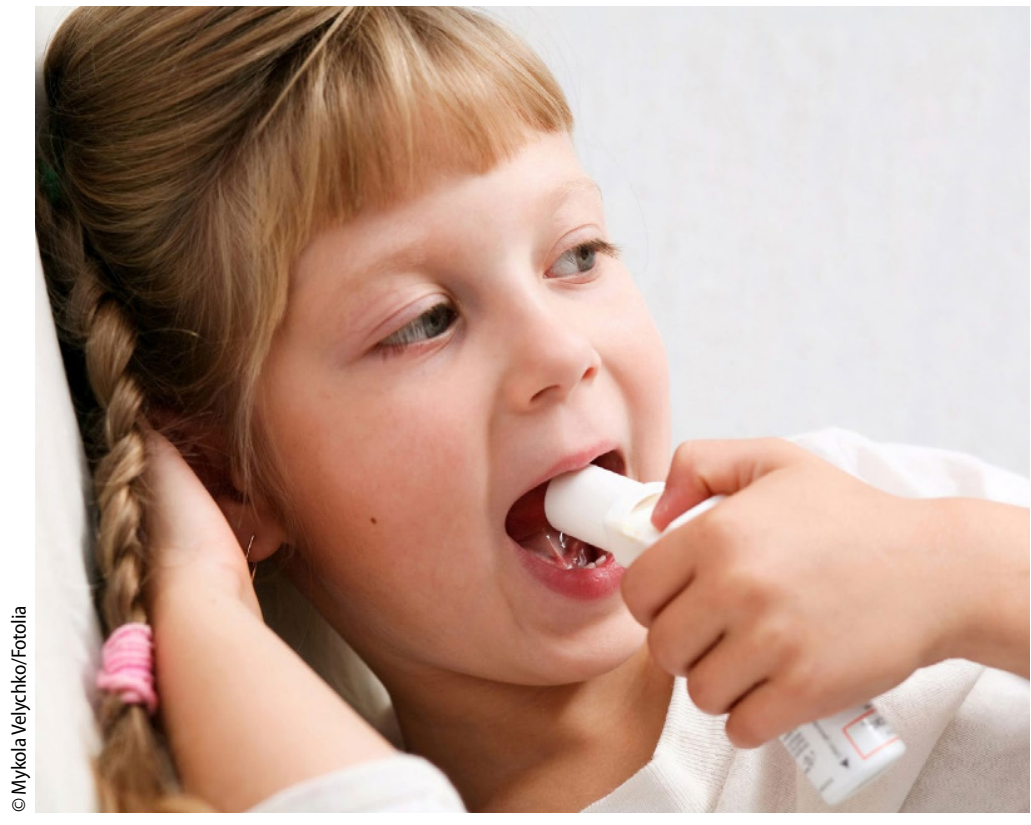

$\Delta$ Dass Kinder mit Asthma bronchiale während der Pandemie mit SARS Cov-2 in den Kindergarten oder in die Schule gehen sollen, steht außer Frage (Symbolbild mit Fotomodell) rungsvideos von folgenden Sponsoren unterstützt: Alk-Abelló, AstraZeneca, Bencard Allergie, Menarini Pharma, Novartis, Sanofi, Thermo Fisher.

\section{Weitere Informationen:}

Österreichische Lungenunion

Obere Augartenstraße 24, 1020 Wien

office@lungenunion.at

www.lungenunion.at

Hinweis des Verlags. Der Verlag bleibt in Hinblick auf geografische Zuordnungen und Gebietsbezeichnungen in veröffentlichten Karten und Institutsadressen neutral.

Paediatr. Paedolog. 2020 · 55:266-267 https://doi.org/10.1007/s00608020-00837-7

(c) Springer-Verlag GmbH Austria, ein Teil von Springer Nature 2020

Hier steht eine Anzeige.

\section{in Springer}

